\title{
Chemical Immunology
}

\section{Vol. 70}

Series Editors Luciano Adorini, Milan

Ken-ichi Arai, Tokyo

Claudia Berek, Berlin

J. Donald Capra, Oklahoma City, Okla.

Anne-Marie Schmitt-Verhulst, Marseille

Byron H. Waksman, New York, N.Y.

KARGER $\begin{aligned} & \text { Basel· Freiburg } \cdot \text { Paris } \cdot \text { London } \cdot \text { New York } \cdot \\ & \text { Nelhi } \cdot \text { Bangkok } \text { Singapore } \cdot \text { Tokyo } \cdot \text { Sydney }\end{aligned}$ 


\section{Immunology of Intracellular Parasitism}

Volume Editors

Foo Yew Liew, Glasgow

Frank E. G. Cox, London

17 figures and 6 tables, 1998

KARGER $\begin{aligned} & \text { Basel·Freiburg } \cdot \text { Paris } \cdot \text { London } \cdot \text { New York } \\ & \text { New Delhi } \cdot \text { Bangkok } \cdot \text { Singapore } \cdot \text { Tokyo } \cdot \text { Sydney }\end{aligned}$ 


\section{Chemical Immunology}

Formerly published as 'Progress in Allergy'

Founded 1939 by Paul Kallòs

\section{Foo Yew Liew}

Department of Immunology

University of Glasgow

Western Infirmary

UK-Glasgow G11 6NT

(United Kingdom)

\section{Frank E.G. Cox}

Department of Infectious \& Tropical Diseases London School of Hygiene \& Tropical Medicine

Keppel Street

UK-London WC1E 7HT (United Kingdom)

Bibliographic Indices. This publication is listed in bibliographic services, including Current Contents ${ }^{\circledR}$ and Index Medicus.

Drug Dosage. The authors and the publisher have exerted every effort to ensure that drug selection and dosage set forth in this text are in accord with current recommendations and practice at the time of publication. However, in view of ongoing research, changes in government regulations, and the constant flow of information relating to drug therapy and drug reactions, the reader is urged to check the package insert for each drug for any change in indications and dosage and for added warnings and precautions. This is particularly important when the recommended agent is a new and/or infrequently employed drug.

All rights reserved. No part of this publication may be translated into other languages, reproduced or utilized in any form or by any means electronic or mechanical, including photocopying, recording, microcopying, or by any information storage and retrieval system, without permission in writing from the publisher.

C) Copyright 1998 by S. Karger AG, P.O. Box, CH-4009 Basel (Switzerland)

Printed in Switzerland on acid-free paper by Reinhardt Druck, Basel

ISBN 3-8055-6621-2 


\section{Contents}

\section{Preface}

\section{Immunity to Listeria monocytogenes}

R.J. North, J.W. Conlan, Saranac Lake, N.Y.

1 Listeria and Listeriosis

3 Murine Listeriosis as a Model of Cellular Immunity to Infection

4 Limitations of the Adoptive Immunization Assay

6 Resolution of Primary Infection

6 Role of Tissue Macrophages in Initial Defense

7 The Essential Early Role of Neutrophils

9 The Role of NK Cells

11 The Role of T Cells

13 Evidence against a Role for T Cells

14 Immunity to Reinfection

16 Conclusion

17 References

21 Immunity to Mycobacteria with Emphasis on Tuberculosis: Implications for Rational Design of an Effective Tuberculosis Vaccine

S.H.E. Kaufmann, Ulm/Berlin; P. Andersen, Copenhagen

22 Mycobacterial Diseases

23 T Cells as Central Mediators of Protection

24 Mycobacterial Localization and Antigen Presentation

25 CD4 T Lymphocytes 
25 CD8 T Cells

$26 \gamma \delta \mathrm{T}$ Cells

$27 \mathrm{DN} \alpha \beta \mathrm{T}$ Cells

28 Cytokines

32 Cytolytic T Lymphocyte Responses

$33 \mathrm{~T}$ Cells Mediating Immunological Memory

35 Mycobacterial Antigens

37 Mycobacterial Antigens Recognized by T Cells

39 Extracellular versus Somatic Antigens - Early versus Late Antigens

41 The Design of Novel Vaccines against Tuberculosis

42 Subunit Vaccines

44 Recombinant Carriers

44 BCG

45 Other Carriers

45 Attenuated Mycobacterial Strains

46 DNA Vaccines

47 Testing of Experimental Vaccines in Animal Models

48 Acknowledgements

48 References

60 Experimental Cutaneous Leishmaniasis: Induction and Regulation of T Cells following Infection of Mice with Leishmania major

P. Scott, J.P. Farrell, Philadelphia, Pa.

60 Introduction

62 Initiation of the Immune Response to L. major

68 Regulation of Established Immune Responses to L. major

75 Conclusions

76 Acknowledgements

76 References

81 Immunoregulation during Toxoplasmosis

J. Alexander, Glasgow; C.A. Hunter, Philadelphia, Pa.

81 Introduction

82 Host and Parasite Factors Affecting Susceptibility to T. gondii

83 NK Cells and Innate Resistance to T. gondii

$86 \mathrm{~T}$ Cells and the Adaptive Immune Response

87 Parasite Killing and Tachyzoite to Bradyzoite Transformation

89 Toxoplasmosis and the Brain

92 Parasite Control versus Host Pathogenesis

93 Vaccination

95 Conclusion

96 References 
103 Immunological Control of Cryptosporidium Infection

V. McDonald, G.J. Bancroft, London

105 Innate Immune Responses to Cryptosporidium

108 The Role of $\mathrm{T}$ Cells in Response to Cryptosporidial Infection

111 Involvement of IFN- $\gamma$ in T-Cell-Mediated Immunity

113 Production of Other $T$ Cell Cytokines during Infection

115 Antibody Response to Infection

117 Immunotherapy against Cryptosporidiosis

119 Conclusions

119 References

124 Immunology of Trypanosoma cruzi Infections

S.G. Reed, Seattle, Wash.

124 Introduction

124 The Parasite

125 Infection and Disease

127 Immunobiology of T. cruzi Infection

127 Experimental Models

128 The Role of T Cells in T. cruzi Infections

130 TGF- $\beta$

133 IL-10

136 Cytokine Responses in Human T. cruzi Infections

139 Autoimmune Responses in Human T. cruzi Infection

140 Practical Considerations

141 References

144 The Immune Response to Asexual Blood Stages of Malaria Parasites N.C. Smith, A. Fell, M.F. Good, Brisbane

144 Introduction

$146 \mathrm{~T}$ Cells in Resistance to Asexual Blood Stage Malaria

151 Evidence for Involvement of Antibodies in Resistance to Asexual Blood Stage Malaria

155 The Role of the Spleen in Resistance to Malaria

156 Conclusions

157 Acknowledgements

157 References

163 Immunobiology of Infections with Theileria parva in Cattle W.I. Morrison, Compton, D.J. McKeever, Nairobi

163 Introduction

164 Parasite-Host Cell Relationship

165 Features of Immunity 
166 Immune Responses to Sporozoites

169 Immune Responses to Parasitized Lymphocytes

171 Parasite Strain Heterogeneity

172 Parasite Strain Specificity of CTL Responses

174 Immunodominance as a Determinant of Strain Specificity of CTL

176 Immune Responses to Primary Infection

178 Influence of Infected Cell Type on Pathogenesis

180 Concluding Remarks

181 References

186 Immunobiology of African Trypanosomiasis

J.M. Sternberg, Aberdeen

187 Life Cycle

188 Immunity and Antigenic Variation

189 Immunosuppression during Trypanosomiasis

189 Suppressor Macrophage Activity in Trypanosomiasis

191 IFN- $\gamma$ in Trypanosomiasis

193 Mechanisms of Macrophage Activation in Trypanosomiasis

195 Discussion and Conclusions

196 References

200 Author Index

201 Subject Index 


\section{............................. \\ Preface}

The intracellular habitat provides a secure environment for many microorganisms but those that parasitize the cells of the immune system, particularly macrophages and lymphocytes, live on a knife edge between survival and triggering a potentially destructive immune response. All intracellular organisms have, therefore, evolved ways of evading the immune response with the consequence that the infections they cause tend to be long and chronic and accompanied by immune responses that are not necessarily protective and may actually be counter-protective. For many years it was accepted dogma that immunity to such organisms was largely cell-mediated and characterized by the development of delayed-type hypersensitivity (DTH) reactions. A fuller understanding of the actual mechanisms of both survival in the immune host and the protective immune responses evoked was not possible until a decade ago when Tim Mosmann, Robert Coffman and their colleagues showed that cell-mediated responses resulted from the activities of cytokines produced by Th1 CD4 + T lymphocytes and that humoral immune responses were under the control of Th2 CD4 + cells. This provided a framework within which the various components of the immune response, particularly to infectious organisms, could be analysed. Over the past decade, there have been a number of additions and modifications to this overall pattern, including the findings that CD8 + T cells have similar properties to CD4 + cells. However, the basic dichotomy still holds and it is now generally held that clear-cut Th1-type and Th2-type responses represent polar positions and that, in most infections, it is the actual balance between Th1 and Th2 cytokines that determines the overall pattern and outcome of any particular immune response.

Central to our understanding of the immune response to intracellular parasites is the role of IFN- $\gamma$ in macrophage activation culminating in the release of toxic reactive oxygen radicals (ROI), nitric oxide (NO) and tumor necrosis factor (TNF) that separately or together are responsible for the de- 
struction of many intracellular parasites. IFN- $\gamma$ is also a product of natural killer (NK) cells under the influence of IL-12. The overall immune response in any particular infection is, therefore, the result of the activities of a number of cytokines some of which drive protective antiparasitic immune responses and some of which are counter-protective and are induced by the parasite itself in order to manipulate the immune response in such a way as to ensure its own survival. This overall pattern is common to all infections caused by intracellular parasites and this volume explores both these common features and also the special features whereby particular parasites evade immune attack and how they are destroyed. An understanding of the roles of lymphocytes, macrophages, NK cells and cytokines in the immune response is essential for the development of vaccines, for strategies for ameliorating immunopathological responses and for explaining why some infections are important concomitants in AIDS while others are not.

The organisms discussed in this volume include prokaryotes and eukaryotic protozoa and these will be used to illustrate and expand on the principles outlined above. Listeriosis has long been regarded as the prototype of DTH (Th1) responses but this situation is no longer as clear cut as it was once thought to be and there is now evidence that CD8 + lymphocytes, macrophages, neutrophils and NK cells are also involved, the last three in the absence of T lymphocytes. In mycobacterial infections the essential role of Th1 CD4+ cells is not in doubt but there is increasing evidence that CD8 + cells and IL-12 are also involved. Turning to the protozoa, cutaneous leishmaniasis is usually regarded as a paradigm of the Th1/Th2 dichotomy with CD4 + Th1 products having a protective role and $\mathrm{Th} 2$ products being counter-protective. This remains the case but, in addition, IL-12 and CD8 + cells are now known to be involved in protection and the outcome of the infection is determined by the balance between the Th1 and Th2 cytokines. In toxoplasmosis, more than any other protozoan infection, the Th1/Th2 balance is crucial in determining whether the outcome is chronic or acute and during the infection there is a swing from the production of protective cytokines to the production of regulatory cytokines that prevent the development of pathology.

Cryptosporidiosis is an emerging infection particularly in AIDS patients and here, as in most other intracellular infections, CD4 + Th1 cells predominate in the immune response particularly during the early phases of the infection but NK cells and IL-12 also play minor but significant roles. In Chagas disease, caused by Trypanosoma cruzi, the immune response is complicated by the fact that there is immunodepression early in the infection but later there is an autoimmune component and, although IFN- $\gamma$-activated macrophages are involved in parasite killing, this is inhibited by other cytokines. Malaria is the most complicated of all the infections discussed here as the infection comprises 
a number of antigenically distinct phases, some intracellular and some extracellular, each evoking a specific immune response. In the erythrocytic stages of the infection antibodies play a major role in protection but the role of macrophages is ambiguous as there is evidence that macrophage products such as NO and TNF are both protective and involved in the disease process. In theileriosis, the only wholly veterinary infection discussed here, the parasites live in lymphocytes and this is the only protozoal infection in which immunity is mainly effected by CD8 + CTL cells and is class I restricted. Finally, African trypanosomiasis is different from the other parasites described here in that the infection is extracellular and immunity is largely antibody-mediated. It is included here for comparison because the overall picture is one in which evasion of the immune response results from antigenic variation but there is also evidence that Th1 cytokines are manipulated by parasite factor(s) to permit parasite survival. In addition, macrophages are instrumental in immunosuppression, $\mathrm{NO}$ is infection-enhancing and IFN- $\gamma$ stimulates parasite growth.

The purpose of this volume is to explore the immune responses to a selection of important intracellular parasites in order to stimulate interest in a comparative approach from which lessons concerning the development of vaccines, ameliorating deleterious immune responses and preventing the complications of concurrent infections with HIV can be learned.

Foo Yew Liew, Glasgow

Frank E.G. Cox, London 